\title{
Super Barrier Rectifier-A New Generation of Power Diode
}

\author{
Vladimir Rodov, Alexei L. Ankoudinov, and Taufik, Senior Member, IEEE
}

\begin{abstract}
The main principle behind the new super barrier rectifier (SBR) approach is to create the "super" barrier for majority carriers without unreliable metal-semiconductor Schottky contact. The SBR technology creates such barrier in the MOS channel. The height of this barrier can be easily adjusted by the doping concentration in the channel. This paper demonstrates that the new power diodes combine high performance and high reliability for low voltage applications (below $100 \mathrm{~V}$ ). The underlying concepts and analysis of operation are presented as well as the laboratory test results that compare performance and reliability between Schottky and the new SBR diode.
\end{abstract}

Index Terms-Power semiconductor diodes, rectifiers, semiconductor devices, semiconductor diodes.

\section{INTRODUCTION}

$\mathbf{T}$ HE PERFORMANCE and reliability of power diodes often limit the design of modern power devices. Schottky diode is the dominant technology for rated voltage below $250 \mathrm{~V}$. Schottky diodes are attractive to power electronic applications due to their high performance: low forward voltage $\left(V_{F}\right)$ at reasonable leakage current $\left(I_{R}\right)$ and small reverse recovery times $\left(t_{\mathrm{RR}}\right)$ [1]. Schottky technology is well known for its relatively high level of leakage current in OFF state compared to $p$-n diodes. The leakage in traditional Schottky diodes does not stay constant, but increases exponentially with the applied bias due to the image charge potential barrier lowering [1]. The main problem of Schottky diodes is the low reliability of metal-semiconductor contact, which limits their ability to operate at high temperatures, and reduces capability to withstand forward and reverse surges. Schottky diodes rapidly lose their performance and reliability with temperature, thus requiring additional consideration of heat removal.

The alternative $p-n$ junction technology in comparison provides lower performance but higher reliability. The p-n junction diodes typically have larger $V_{F}$ and $t_{\mathrm{RR}}$. Being a minority carrier device, the p-n diode has larger reverse recovery time than Schottky [1]. Lifetime control methods allow reduction of recovery time at the expense of the increase of the forward voltage. Despite these disadvantages, $\mathrm{p}$-n diodes dominate rectifier mar-

Paper IPCSD-07-055, presented at the 2007 IEEE Applied Power Electronics Conference and Exposition, Anaheim, CA, February 25-March 1, and approved for publication in the IEEE TRANSACTIONS ON INDUSTRY APPLICATIONS by the Power Electronics Devices and Components Committee of the IEEE Industry Applications Society. Manuscript submitted for review December 20, 2006 and released for publication August 16, 2007.

V. Rodov and A. L. Ankoudinov are with Lakota Technologies, Inc., Seattle, WA 98052 USA (e-mail: vrodov@hotmail.com; ankoudinov@comcast.net).

Taufik is with the Electrical Engineering Department, California Polytechnic State University, San Luis Obispo, CA 93407 USA (e-mail: taufik@calpoly. edu).

Digital Object Identifier 10.1109/TIA.2007.912752

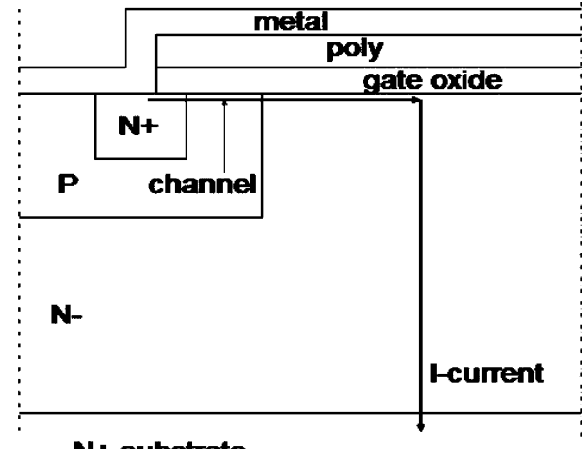

N+ substrate

Fig. 1. Cross section of the SBR cell.

ket when high breakdown voltage or high reliability is required, e.g., at high operation temperatures or high currents.

Previously, the channel diode technology [2], [3] has been suggested to combine high performance with high reliability, but it did not provide viable competition to Schottky diodes. This paper introduces new super barrier rectifier (SBR) technology, which has both high performance and high reliability, a combination which cannot be achieved with Schottky or p-n junction technologies. We discuss the operation of SBR diodes with rated voltage $100 \mathrm{~V}$ and lower. The SBR operation at higher rated voltage is complicated due to carrier density modulation, which will be addressed in separate publication. We also demonstrate that real SBR diodes can readily compete with Schottky for a number of practical applications.

\section{SBR STRUCTURE AND OPERATION PRINCIPLES}

The SBR diode overcomes the aforementioned issues with Schottky and p-n diodes by using the MOS structure as illustrated in Fig. 1. The most important part of the structure is the MOS channel formed under the thin gate oxide, where the "super" barrier for majority carriers is created without unreliable Schottky contact. The "super" barrier guarantees that forward bias performance will be similar to Schottky with higher reliability. Moreover, the SBR technology improves reverse bias performance. The absence of image charge potential barrier reduction results in constant leakage (current limiting function) and abrupt breakdown (good for voltage regulation and protection). In order to make the SBR an effective rectifier, the channel has to be very short (approximately $0.2 \mu \mathrm{m}$ [4]). The $\mathrm{n}^{+}$contact region is introduced to ensure ohmic contact with the metal.

The transient behavior of the SBR is similar to Schottky for devices rated below $100 \mathrm{~V}$. The injection of minority carriers is not involved in this case, since it can occur at the $p$-n junction of 


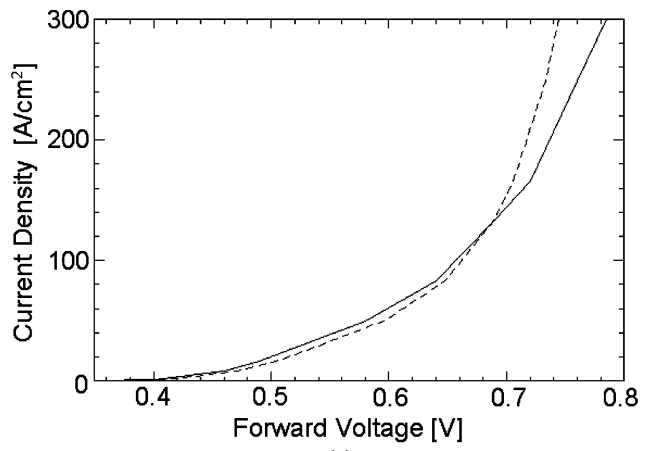

(a)

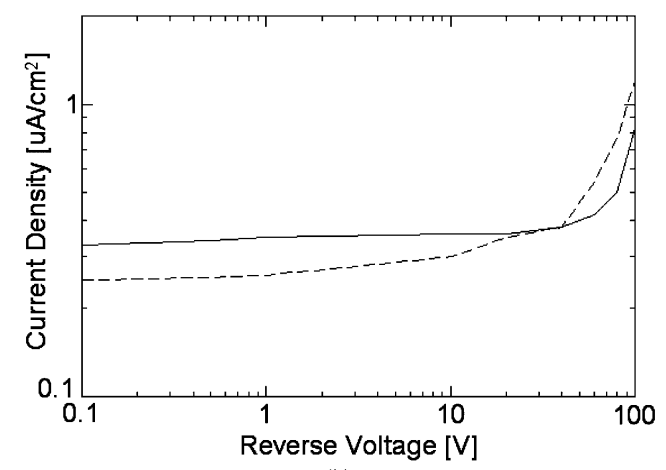

(b)

Fig. 2. (a) Forward $\left(\mathrm{A} / \mathrm{cm}^{2}\right)$ and (b) reverse $\left(\mu \mathrm{A} / \mathrm{cm}^{2}\right)$ current density versus applied voltage: simulation (dashed) versus measured (solid).

the SBR structure only when applied forward bias is above $0.6 \mathrm{~V}$ [5]. Such injection is important for high forward surge capability. For the forward bias, the depletion layers of $p$ - $n$ junctions in the region between the channels do not overlap and allow the current to flow through the $\mathrm{n}^{-}$region. The particular concentrations and dimensions of various regions can be adjusted for desired diode behavior. The thickness and donor concentration of the $\mathrm{n}^{-}$region control the maximum operation voltage. The acceptor concentration in $\mathrm{p}$ region controls the barrier height and the reverse leakage current in such a way that one can trade between low forward voltage and low reverse current [4].

The $\mathrm{p}-\mathrm{n}$ diodes in parallel to the channel are essential for keeping the leakage current small. In reverse bias regime, when applied reverse voltage reaches a few hundred millivolts, the pinch-off effect takes place due to overlap of these depletion layers. At this point, reverse leakage current through MOS channels changes much slower with the increase of reverse voltage. At higher reverse biases, the depletion regions spread into $\mathrm{n}^{-}$ region and into $\mathrm{p}$ regions of the $\mathrm{p}-\mathrm{n}$ junctions. This increases a generation current contribution to the reverse leakage.

\section{TESTS AND MEASUREMENTS}

In order to test the aforementioned operation principles, we performed computer simulations with Integrated Systems Engineering Technical Computer-Aided Design (ISE TCAD) software [6], and compared the results to the measurement on the real SBR device. Fig. 2(a) shows such comparison for the SBR diode with $100 \mathrm{~V}$ rated voltage. It shows a very good agreement between simulation result (dashed line) and real measurement
TABLE I

PERFormance AND RELIABILITY COMPARISON OF 20 A 100 V Diodes

\begin{tabular}{|l|c|c|c|}
\hline $\begin{array}{l}20 \mathrm{~A} 100 \mathrm{~V} \\
\text { Diode }\end{array}$ & $\begin{array}{c}\text { APD } \\
\text { SBR20U100 }\end{array}$ & $\begin{array}{c}\text { ST } \\
\text { STPS20H100 }\end{array}$ & $\begin{array}{c}\text { Vishay } \\
\text { MBR20100 }\end{array}$ \\
\hline Typical VF $(\mathrm{V})$ & 0.65 & 0.71 & 0.72 \\
\hline IR (typ) $(\mu \mathrm{A})$ & 35 & 1 & 0.02 \\
\hline IRRM $(\mathrm{A})$ & 3 & 1 & 0.5 \\
\hline EAS (mJ) & 205 & 120 & 24 \\
\hline $\begin{array}{l}\text { Thermal } \\
\text { Runaway (ㅇ) }\end{array}$ & 225 & 205 & 160 \\
\hline $\begin{array}{l}\text { IFSM (A) } \\
\text { (square, 10 ms) }\end{array}$ & 285 & 230 & 170 \\
\hline
\end{tabular}

TABLE II

Performance Comparison of 1 A 30 V Diodes in Sod-323 PACKAGE

\begin{tabular}{|c|c|c|c|c|}
\hline 1A 30V Diode & $\begin{array}{c}\text { APD } \\
\text { SBR130 }\end{array}$ & Philips & $\begin{array}{c}\text { Toshiba } \\
\left(\text { Low } I_{R}\right)\end{array}$ & $\begin{array}{c}\text { Toshiba } \\
\left(\text { Low } V_{F}\right)\end{array}$ \\
\hline$V_{F}$ (typical) $[V]$ & 0.39 & 0.455 & 0.47 & 0.4 \\
\hline$I_{R}$ (typical) $[\mu \mathrm{A}]$ & 15 & 20 & 10 & 500 \\
\hline
\end{tabular}

(solid line). At current density of $140 \mathrm{~A} / \mathrm{cm}^{2}$, both results show a forward voltage $V_{F}=0.7 \mathrm{~V}$. The discrepancy at higher voltages is due to the voltage drop on wires outside the actual SBR diode.

The behavior of SBR for reverse bias is shown in Fig. 2(b), which demonstrates a steady leakage up to breakdown voltage. The potential barrier lowering due to the image charge, which is essential for Schottky diode, is absent in the SBR. The SBR reverse current is typical of p-i-n diode, where reverse current consists of the constant injection and growing ionization currents. As an example, the actual $10 \mathrm{~A} 100 \mathrm{~V}$ SBR diode with $V_{F}=$ $0.72 \mathrm{~V}$, the leakage current was found to be less than $50 \mathrm{nA}$, outperforming Schottky diodes that have much higher leakage for similar forward voltage.

To demonstrate the performance and reliability of the SBR diode, it is compared to Schottky diodes of comparable ratings and package size (TO-220) in Table I. The SBR diode combines very high performance $\left(V_{F}\right.$ and $I_{R}$ are similar or better than Schottky) with much better reliability parameters (maximum reverse repetitive surge current $I_{\mathrm{RRM}}$, maximum energy during avalanche surge $\left(E_{\mathrm{AS}}\right)$, thermal runaway temperature, and forward surge current $\left.I_{\mathrm{FSM}}\right)$.

The comparison of 1 A $30 \mathrm{~V}$ devices in SOD-323 typical for cell phone and other handheld applications is provided in Table II. Some applications need diodes with low leakage and others require low $V_{F}$. Thus, Toshiba and other companies make two types of 1 A $30 \mathrm{~V}$ diodes. The SBR diode has both low leakage and low $V_{F}$, thus demonstrating the high performance advantage of the SBR technology over Schottky for low breakdown voltages.

To put the SBR diode into a real circuit test, a $12 \mathrm{~V} 30 \mathrm{~W}$ buck converter was designed and built to show its performance compared with that of a Schottky diode of the same voltage and current ratings $(20 \mathrm{~A}, 40 \mathrm{~V})$ and package size (TO-220). The laboratory measurements show that the overall efficiency of the buck converter with the SBR is overall better than that using comparable Schottky. Fig. 3 depicts that the overall converter's 


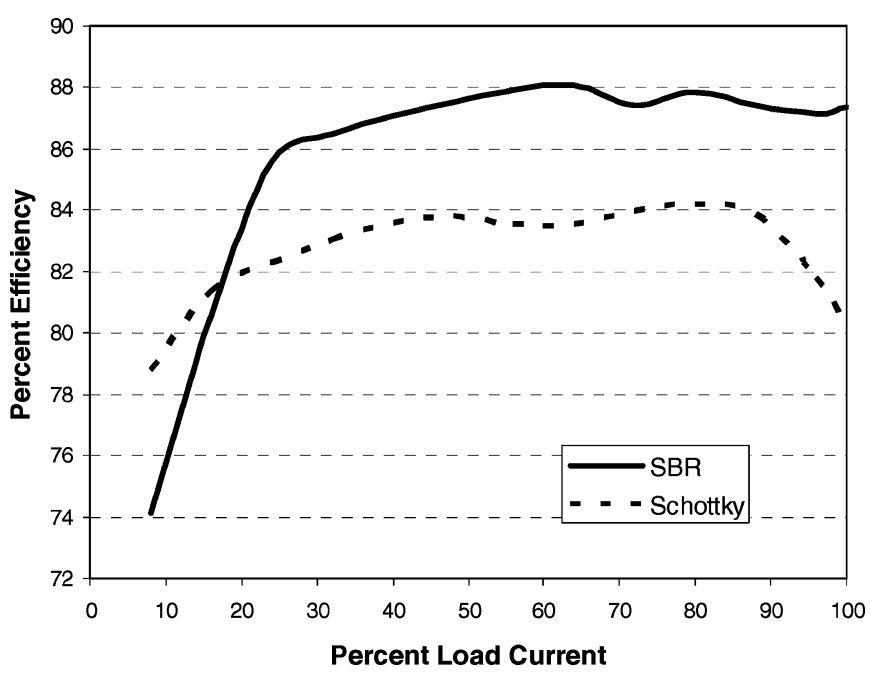

Fig. 3. Buck converter's efficiency with SBR (solid) and Schottky (dashed).

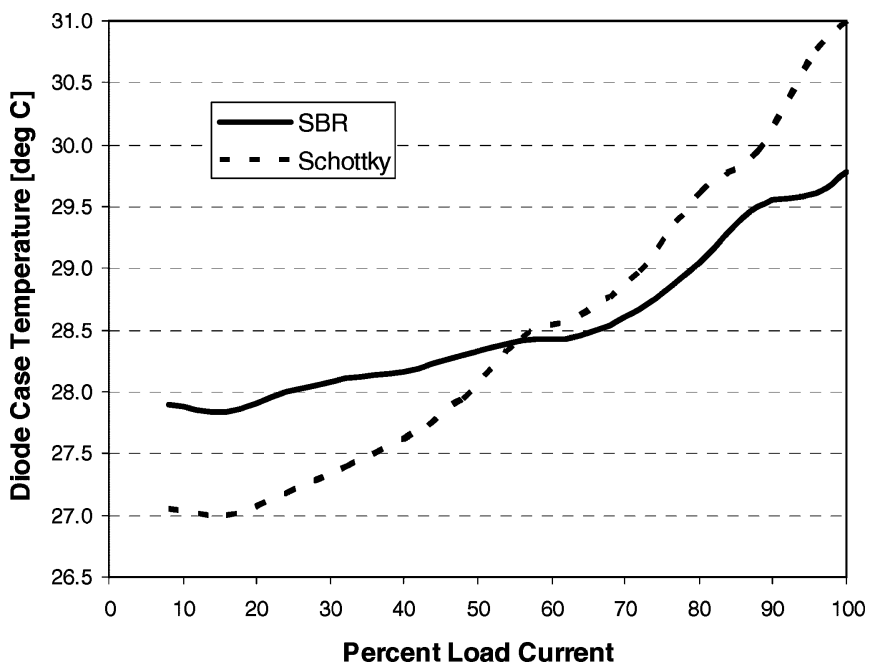

Fig. 4. Case temperature measurements of SBR (solid) versus Schottky (dashed).

efficiency is improved with the SBR throughout loading conditions higher than about $16 \%$. As shown in Fig. 3, efficiency improvement varies up to $6 \%$ at full load current.

Furthermore, diode case and MOSFET case temperature measurements using the same $30 \mathrm{~W}$ buck converter were conducted. As shown in Fig. 4, the case temperature of the SBR has a more gradual slope than that of Schottky. It is interesting to note that for this particular converter operated above 55\% loading, the SBR diode is cooler than the Schottky. At $10 \%$ load for example, the Schottky is about $0.9^{\circ} \mathrm{C}$ cooler than the SBR. However, at $100 \%$ load, the SBR is actually cooler by about $1.25^{\circ} \mathrm{C}$.

The difference in performance between the SBR and Schottky becomes more noticeable when the results of MOSFET's case temperature measurements are plotted, as shown in Fig. 5. Here, clearly, the SBR outperforms Schottky under most of the loading conditions. Beyond about $15 \%$ loading, the MOSFET runs cooler with the SBR than with Schottky. This further accentuates the benefit of SBR's softer and more stable recovery than that of Schottky.

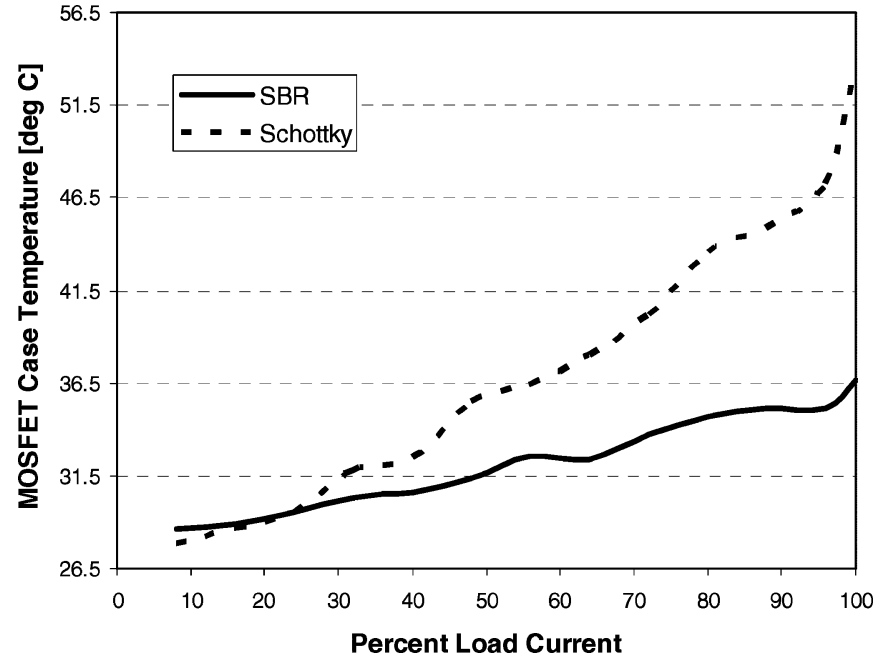

Fig. 5. MOSFET's case temperature measurements when the converter is using SBR (solid) versus Schottky (dashed).

\section{SUMMARY}

The SBR demonstrates attractive properties as a practical power diode. The SBR diodes show a combination of good performance with good reliability. The forward bias behavior for the low voltage devices is similar to that of the Schottky diode. This is expected since by construction, the SBR channel region serves as a potential barrier for the majority (electron) carriers. The barrier height can be tuned for particular application in SBR diodes, while in Schottky diodes, it is fixed to the metals that make good contact with silicon. The SBR diodes typically have lower leakage current compared with the Schottky for the same forward characteristic, due to the pinch-off effect and absence of image charge potential barrier reduction. Thus, the SBR performance is distinctively better than Schottky rectifiers for rated voltage below $100 \mathrm{~V}$. The SBR technology can be used to make diodes in smaller packages or reduce the supply chains. The SBR diode's performance is similar to Schottky, but its reliability is much better $\left(20^{\circ} \mathrm{C}\right.$ higher thermal runaway, three times higher reverse surge capability, which is essential for protection). These advantages of the SBR technology permit the development of practical devices that can successfully compete with existing Schottky or p-n junction diodes for a number of practical applications based on better performance or better reliability.

\section{REFERENCES}

[1] S. M. Sze, Physics of Semiconductor Devices. New York: Wiley, 1983.

[2] Q. Huang and G. A. J. Amaratunga, "MOS controlled diode, a new power diode," Solid State Electron., vol. 38, pp. 977-980, 1995.

[3] H. P. Yee, P. O. Lauritzen, and S. S. Yee, "Channel diode, a new fast switching power diode," in Proc. 1992 ISPSD, pp. 72-79.

[4] P. Chang, G.-C. Chern, W. Y. W. Hsueh, and V. Rodov, "Method of fabricating power rectifier device to vary operating parameters and resulting device," U.S. Patent 6448160, Sep. 10, 2002.

[5] C. T. Sah, R. N. Noyce, and W. Shockley, "Carrier generation and recombination in p-n junctions and p-n junction characteristics," in Proc. IRE, 1957, vol. 45, pp. 1228-1243.

[6] ISE TCAD User Manual, ISE Integrated Systems Engineering AG, Zurich, Switzerland, 2000. 


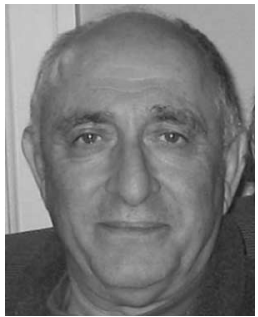

Vladimir Rodov received the M.S. degree in electronic engineering from Moscow Power Engineering Institute, Moscow, Russia, in 1966, the Ph.D. degree in solid-state electronics from the Postgraduate School of the Moscow Electrotechnical Institute, Moscow, in 1968, and the M.S. degree (with Hons.) in mathematics from Moscow State University, Moscow, in 1969.

He was a Senior Engineer at the International Rectifier Corporation. He was also a Senior Scientist at TRW Semiconductors where he led a broad range of projects spanning from microwave to power devices, and developed and implemented an enabling technology (rapid thermal processing), which allowed production of super high-frequency microwave Si bipolar transistors (U.S. patent 4610730). He also led the development of, and patented the methodology for, the manufacturing of monomolecular films for applications in electronics and medical industries at the Molecular Electronics Corporation (MEC). He was with APD Semiconductor (now Diodes, Inc.), where until November 2006, he had been the Chief Technical Officer. He is the author or coauthor of more than 50 published scientific papers, has edited two books, and holds over 20 U.S. patents in the areas of semiconductor devices and monomolecular films.

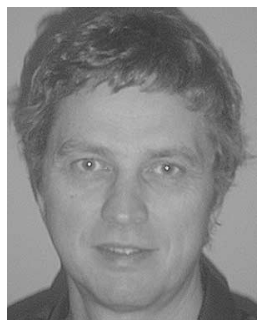

Alexei L. Ankoudinov received the M.S. degree in physics from St. Petersburg State University, St. Petersburg, Russia, in 1987, and the Ph.D. degree in physics from the University of Washington, Seattle, in 1996.

He was with APD Semiconductor (now Diodes, Inc.), where until November 2006, he was an R\&D Manager, and was engaged in all aspects of design, manufacturing, and testing of super barrier rectifier (SBR) diodes. He was an Assistant Professor in the Solid State Theory Group, Physics Department, University of Washington. He was also involved in the topic of interaction between $\mathrm{X}$-rays and condensed matter. He is the coauthor of the FEFF software that helps researchers to obtain structural, electronic, and magnetic information about materials on atomic scale. He is the author or coauthor of more than 50 published scientific papers.

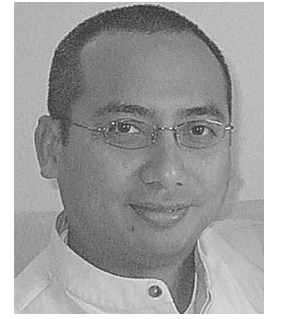

Taufik (S'97-M'99-SM'07) was born in Jakarta, Indonesia, in 1969. He received the B.S. degree in electrical engineering from the Northern Arizona University, Flagstaff, in 1990, the M.S. degree in electrical engineering from the University of Illinois, Chicago, in 1993, and the Dr. Eng. degree from Cleveland State University, Cleveland, $\mathrm{OH}$, in 1999.

He is currently an Associate Professor in the Electrical Engineering Department, California Polytechnic State University, San Luis Obispo. His current research interests include the area of switched mode dc-dc converters. 